\title{
The structure of water around the compressibility minimum.
}

\author{
Authors: L.B. Skinner ${ }^{1,2}$ C.J. Benmore ${ }^{1, *}$, J.C. Neuefeind ${ }^{3}$, J.B. Parise $2,4,5$ \\ Affiliations: \\ ${ }^{1}$ X-ray Science Division, Advanced Photon Source \\ Argonne National Laboratory, Argonne, IL 60439, USA. \\ 2 Mineral Physics Institute, Stony Brook University, \\ Stony Brook, New York, NY 11794-2100, USA. \\ 3 Spallation Neutron Source, Oak Ridge National Laboratory, \\ Oak Ridge Tennessee, 37922. \\ 4 Department of Geosciences, Stony Brook University, \\ Stony Brook, New York, NY 11794-2100, USA. \\ 5 Photon Sciences Division, Brookhaven National Laboratory, Upton, NY 11973, USA. \\ *Correspondence to: benmore@aps.anl.gov
}

\begin{abstract}
.
Here we present diffraction data that yields the oxygen-oxygen pair distribution function, $g_{o o}(r)$, over the range -20 to $+93^{\circ} \mathrm{C}$. The running $\mathrm{O}-\mathrm{O}$ coordination number, which represents the integral of the pair distribution function as a function of radial distance, is found to exhibit an isosbestic point at 3.30(5) $\AA$. The probability of finding an oxygen atom surrounding another oxygen at this distance is therefore shown to be independent of temperature and corresponds to an O-O coordination number of 4.3(2). Moreover, the experimental data also shows a continuous transition associated with the second peak position in $g_{o o}(r)$, concomitant with the compressibility minimum at $46.5^{\circ} \mathrm{C}$.
\end{abstract}


Generally liquids contract and become less compressible upon cooling, water, however, becomes more compressible below $46.5^{\circ} \mathrm{C}{ }^{1}$. Here we show this well-known phenomenon takes place in the same temperature region as continuous structural changes in the second nearest neighbor oxygen-oxygen (O-O) peak of the pair distribution function, obtained through the measurement of high energy x-ray diffraction data out to high momentum transfers $(Q)$. The structure of water and molecular arrangements in water at distances beyond the first nearest neighbor has been debated since the first $\mathrm{x}$-ray measurements were made and this continues to the present day ${ }^{2-14}$. In addition, the density dependent O-O network topology of water has been found to be similar to that of tetrahedral liquids $\mathrm{Si}$ and $\mathrm{Ge}{ }^{15}$. Our measurements show that the $1^{\text {st }}$ neighbor O-O peak has a constant coordination of 4.3(2) at atmospheric pressure and follows a linear expansion from $-19^{\circ} \mathrm{C}$ to $93^{\circ} \mathrm{C}$, which severely constrains any structural model imposed on liquid water.

Measuring an accurate pair distribution function for water using x-ray diffraction to test different models is not a trivial challenge, since the scattering intensity becomes exceedingly small at high- $Q$. Another fundamental difficulty in defining an exact $\mathrm{O}-\mathrm{O}$ coordination number for water arises because the minimum after first peak in the O-O pair distribution function does not reach zero. The presence of molecules in this region introduces arbitrariness, ambiguity and a strong cut-off dependence into the $\mathrm{O}-\mathrm{O}$ coordination number. Previous studies suggest that the $\mathrm{O}-\mathrm{O}$ coordination number lies between 4 and $5^{16}$, which is in good agreement with our latest efforts to benchmark the oxygen-oxygen pair distribution function at ambient temperature ${ }^{17}$.

We have applied high energy x-ray diffraction to measure the structure of water over the temperature range $4{ }^{\circ} \mathrm{C}$ to $93^{\circ} \mathrm{C}$. This range has been extended using literature data measured at the same beamline covering 19 to $-19^{\circ} \mathrm{C}{ }^{18}$. The high energy $\mathrm{x}$-ray diffraction measurements were made using the beamline 11-ID-C at the Advanced Photon Source (Argonne National Laboratory, USA). Monochromatic x-rays with an incident energy of 114.76(1) keV were detected with an amorphous silicon area detector (Perkin Elmer XRD1621), over three independent experimental runs. The first run used a container-less flowing water stream, the second used water contained in a thin-walled (100 micron) $\mathrm{SiO}_{2}$ glass capillary, and the third used acoustically levitated water droplets 18. Care was taken in the setup to optimize the signal to background ratio and to balance Q-resolution vs. Q-range. For the water stream and capillary runs, scattering measurements were taken using multiple detector positions which were offset perpendicular to the incident beam. This side-stepping ensured any residual effects from pixel-to-pixel response variation in the area detector were minimized. In both the water stream and capillary measurements, temperature was monitored via a thermocouple submerged immediately above or below the incident x-ray beam. In the water stream setup the water reservoir was heated using a hot plate, or cooled using ice. The measurements made on water in a capillary were heated using a hot air gun. The capillaries used were $\sim 3 \mathrm{~mm}$ diameter with a wall thickness $\sim 0.1 \mathrm{~mm}$, further details of the experiments and data analysis are given in ${ }^{17,19,20}$. After careful account of all the necessary corrections, O-O structure factors up to $Q$ values of $\sim 24 \AA^{-1}$ with high statistical precision and low systematic error are presented in Fig. 1A. 
Neutron diffraction Measurements were performed on deuterated $\mathrm{D}_{2}{ }^{18} \mathrm{O}$, and $\mathrm{D}_{2}{ }^{16} \mathrm{O}$ water samples (Cambridge isotopes, UK) sealed under dry nitrogen in thin walled (100 micron) $\mathrm{SiO}_{2}$ glass tubes. These were then mounted above a Nitrogen cryostream (Oxford instruments, UK) on the Nanoscale Ordered MAterials Diffractometer (NOMAD) at the Spallation Neutron Source (SNS), Oak Ridge National Laboratory, USA. The $\mathrm{D}_{2}{ }^{18} \mathrm{O}-\mathrm{D}_{2}{ }^{16} \mathrm{O}$ neutron difference $\left(\Delta \mathrm{S}_{\mathrm{N}}(\mathrm{Q})\right)$ patterns were then measured and analyzed using standard data analysis procedures ${ }^{19,21}$. These $\Delta \mathrm{S}_{\mathrm{N}}(\mathrm{Q})$ patterns consist of $0.69 \cdot \mathrm{S}_{\mathrm{OD}}(\mathrm{Q})+0.31 \cdot \mathrm{S}_{\mathrm{OO}}(\mathrm{Q})$, and zero $\mathrm{S}_{\mathrm{DD}}(\mathrm{Q})$, providing a good guide to the $\mathrm{S}_{\mathrm{OH}}(\mathrm{Q})$ structure factor in water. Example neutron difference patterns at $4^{\circ} \mathrm{C}$ and $75^{\circ} \mathrm{C}$ are shown in 19 .

In addition, the ambient to supercooled water data measured by Neuefeind et al. ${ }^{18}$ was reanalyzed in the present paper using an identical analysis procedure as the other two experimental runs. The data quality of the acoustically levitated measurements is however, slightly lower since no detector side-stepping was used, and the small droplet size limits the signal-to-background ratio. In all x-ray experiments the water used was deionized ultra-filtered grade from Fischer Chemical (Pittsburg, PA, USA). Additional xray data reduction details are given in ${ }^{17,19,20}$.

These high- $Q$ x-ray data have yielded high-resolution pair-distribution functions over a wide range of temperatures using the procedure previously described by Skinner et al. ${ }^{17}$. To extract the dominant $\mathrm{O}-\mathrm{O}$ function the minority $\mathrm{O}-\mathrm{H}$ and $\mathrm{HH}$ terms were subtracted from the measured x-ray data. The room temperature intermolecular $H_{O H}(Q)$ contribution was obtained from averaging three independent sets of neutron scattering measurements and simulation results ${ }^{21,22,23}$ which were all in reasonable agreement ${ }^{17}$. The effect of temperature was included by adding the small temperature difference $\left(\Delta H_{O H}(Q)\right)$ between $H_{O H}(Q)$ at the measured temperature and that at room temperature calculated from TIP4P ${ }^{23}$ molecular dynamics simulations. The inter-molecular $H_{H H}(Q)$ contribution, which has an extremely small weighting in the x-ray measurements, was also estimated and subtracted using TIP4P calculations at each measured temperature. Consequently, the extracted O-O pair distribution $\left(g_{o o}(r)\right)$ functions represent the intrinsic width and height of the first O-O peak, with negligible $(<1 \%)$ additional Fourier transform broadening (see Fig. 1B).

The $1^{\text {st }}$ and $2^{\text {nd }}$ O-O peak maximum positions in $g_{o o}(r)\left(r_{1}, r_{2}\right)$ were obtained by fitting a Gaussian curve locally around the peak maxima (shown in the inset of Fig. 2A). The fit ranges for these curves were $2.65<r(\AA)<2.9$, and $3.9<r(\AA)<5.1$. The error bars given in figure 2A and 2C were calculated from the standard error in each fit. The $r_{1}$ vs. temperature slope of this work $\left(0.000320(18) \AA^{\circ} \mathrm{C}^{-1}\right)$ is slightly lower than that observed in ${ }^{18}$ as the latter, used a fixed peak area and a wider peak fitting range. The wider peak fit range used in ${ }^{16}$ was found to provide better peak width information at the expense of a poorer fit to the position of the maximum in $g(r)$. Fitting only around the peak maxima, as performed in this work is, was used to closely follow the $g(r)$ at the peak, since only the peak position was of interest. The non-linear second neighbor peak 
behavior observed in the pair distribution functions is also reflected in the position of the $2^{\text {nd }}$ peak in the measured $x$-ray structure factors.

The running O-O coordination number, $n_{O o}\left(r_{c u t}\right)$, plotted in figure $1 \mathrm{C}$ represents the integral of the pair distribution as a function of cutoff distance, $r_{c u t}$, and shows an isosbestic point at $3.3 \AA$, corresponding to the maximum in the isochoric temperature derivatives of Bosio et al. ${ }^{24}$. This isosbestic (crossover) point in $\mathrm{O}-\mathrm{O}$ coordination number, means that the probability of finding an oxygen atom surrounding another oxygen atom up to $3.3 \AA$ away is constant with temperature at ambient pressure. The $r=3.3 \AA$ position of the isosbestic point is also the position of the $1^{\text {st }}$ minimum in $r^{2} g_{O o}(r)$. In both $H_{O O}(Q)$ and $g_{O o}(r)$ there are multiple $Q$ and $r$ isosbestic points where the functions cross-over at all measured temperatures. Since the liquid has a slightly different density at each temperature, however, the running coordination numbers diverge at long distances leaving only one isosbestic point in coordination number at $3.3 \AA$ and increasingly divergent crossover positions at higher- $r$.

A key piece of information put forward in argument of the two state mixture model for the structure of water has been the existence of isosbestic points in the Raman and infra-red spectra ${ }^{25,26}$, suggesting that water may be decomposed into a linear combination of two independent spectra. A contrary interpretation of the spectroscopic data, however, has been presented by Geissler ${ }^{27}$, who suggested that isosbestic points can also be explained by a continuous distribution of local environments. Whilst our diffraction data is consistent with both scenarios, the measured coordination number constraints rule out any models in which the average $\mathrm{O}-\mathrm{O}$ coordination number (out to $3.3 \AA$ ) changes over the temperature range $-19^{\circ} \mathrm{C}$ to $93^{\circ} \mathrm{C}$.

It is important to note that previous measurements by Huang et al. ${ }^{10}$, Skinner et al. ${ }^{17}$ and Neuefeind et al. ${ }^{18}$ do not observe any significant fine structure in their x-ray pair distribution functions. Although Fourier transform artifacts are nearly always present in pair distribution functions obtained from diffraction measurements, their magnitude and frequency depend strongly on the Q-range, statistical noise and systematic errors present in a given data set ${ }^{10,17,18}$. Such Fourier oscillations, previously associated with fine structure in the second neighbor peak of water by other authors, have been minimized in the present work by careful data collection procedures, such as long counting times, using multiple detector positions, and the minimization of backgrounds. This has led to increased accuracy in $g_{o o}(r)$ around the first and second O-O peaks in the present work than has previously achieved for water.

Consequently, the high real-space resolution, low noise and low systematic error of our $H_{O O}(Q)$ and $g_{O o}(r)$ functions has allowed accurate determination of the first and second peak maximum positions $\left(r_{1}, r_{2}\right)$ as a function of temperature (Fig. 2A). The position of the first peak maximum, $r_{1}$, in $g_{o o}(r)$ is found to increase linearly with temperature through the compressibility minimum (Fig. 2B). The position of the second peak maximum, $r_{2}$, however, shows significant deviations from linear behavior. Upon heating to $4^{\circ} \mathrm{C}$ there is a small contraction of $r_{2}$, followed by a linear expansion up to $46.5^{\circ} \mathrm{C}$. Past $46.5^{\circ} \mathrm{C}$ a continuous and more rapid expansion occurs, indicating a subtle but distinct 
change in water structure around the compressibility minimum, within an experimental uncertainty of $\pm 5^{\circ} \mathrm{C}$. This is highlighted in figure 2C showing the $r_{2} / r_{1}$ ratio from the experimental data which spans only a $<1 \%$ change in density ${ }^{1}$ over the $46.5 \pm 5^{\circ} \mathrm{C}$ range. The change in $r_{2} / r_{1}$ ratio is not reproduced by the TIP4P model, which shows a linear behavior over a -20 to $100^{\circ} \mathrm{C}$ temperature range. In addition, a linear temperature dependence of the inter-molecular O-D hydrogen bond distance is obtained from oxygen isotopic substitution neutron diffraction measurements within experimental errors (see Fig. 2B inset).

Further analysis of the $2^{\text {nd }} \mathrm{O}-\mathrm{O}$ neighbor peak was performed by investigating the $\mathrm{O}-\mathrm{O}$ coordination number change versus temperature. The integral of the function $r \cdot d_{o o}(r)=4 \pi \rho r^{2}\left[g_{o o}(r)-1\right]$, in a given $r$ region gives the difference in $\mathrm{O}-\mathrm{O}$ coordination from the bulk average density, $\Delta n_{O o}$ (where $\rho$ is the density in molecules per $\AA^{3}$ ). The $r \cdot d_{O o}(r)$ functions at varying temperatures, and their integrals in the $1^{\text {st }}$ and $2^{\text {nd }}$ O-O peaks, are shown in Fig. 3. The $1^{\text {st }}$ peak integral is constant with temperature, which is a consequence of the isosbestic point in the $n_{O O}(r)$ coordination number. The $2^{\text {nd }}$ neighbor coordination, however, increases on cooling, and has a significantly steeper slope below $40-50^{\circ} \mathrm{C}$, consistent with the $r_{2}$ change observed at the compressibility minimum (Figs. 2A,B). The fact that the minimum after the first peak in the $\mathrm{O}-\mathrm{O}$ pair distribution does not return to zero has previously been associated with nonhydrogen bonded molecules penetrating the $1^{\text {st }} \mathrm{O}-\mathrm{O}$ shell, causing structural distortions and a greater range of possible network topologies ${ }^{7,8}$. Similar local structural arrangements have also been observed in other analogous liquid and amorphous systems 15,28 . In the current measurements, the main structural change within the $1^{\text {st }} \mathrm{O}-\mathrm{O}$ peak on cooling, is found to be a sharpening around $2.8 \AA$, where longer neighbors $(>2.9 \AA)$ are exchanged for shorter, hydrogen bound neighbors in equal amounts.

Above $46.5^{\circ} \mathrm{C}$ water exhibits normal liquid behavior, in that as it heats up the compressibility increases. In this high-temperature region the second peak position in $g_{o o}(r)$ is found to expand rapidly, while the $2^{\text {nd }}$ O-O peak average coordination number is essentially constant. At and below the compressibility minimum, however, water appears to form a more stable open network structure as indicated by the more constant $r_{2} / r_{1}$ ratio. The coordination and intensity of the second $\mathrm{O}-\mathrm{O}$ peak also increase on cooling below $46.5^{\circ} \mathrm{C}$ as the water structure becomes more ordered, taking on a more tetrahedral network-like topology. In comparison, the compressibility of hexagonal ice decreases with decreasing temperature, unlike that of supercooled water which increases $^{29}$. So, even though the first two O-O peak positions in $g_{o o}(r)$ contract as water is cooled, it appears that an increasing number of molecules in the second neighbor peak of water are able to expand into empty spaces within the disordered network more easily than those trapped in the rigid crystal lattice, which is $~ 8 \%$ less dense than liquid water. This compressibility minimum temperature has been found to be invariant with increasing pressure, and coincides with a crossover point, where thermal expansion is found to be constant with increasing pressure ${ }^{30}$. In future, it will be interesting to investigate whether the temperature-independent coordination remains at non-ambient pressures, particularly negative pressure, and whether or not the position of the second O$O$ peak tracks the temperature of the compressibility minimum at high and low pressures. 


\section{Acknowledgments:}

Thanks to Rick Spence for support with beamline equipment at the Advanced Photon Source. This work was supported by the U.S. DOE Grant Numbers DE-FG0209ER46650 which supported the experiments, MD simulations, data analysis and manuscript preparation (LBS and JBP), and DE-AC02-06CH11357 for the Advanced Photon Source, Argonne National Laboratory.

\section{References:}

1. G.S. Kell. J. Chem. Eng. Data 20, 97-105 (1975).

2. J.D. Bernal and R.H. Fowler. J. Chem. Phys. 1, 515 (1933).

3. H.E. Stanley, S.V. Buldyrev, M. Canpolat, M. Meyer, O. Mishima, M.R. Sadr-Lahijany, A. Scala, F.W. Starr. Physica A 257, 213-32 (1998).

4. $\quad$ P.G Debenedetti. J. Phys.: Condens. Mat. 15, R1669-R1726 (2003).

5. $\quad$ Ph. Wernet et al. Science. 304, 5673-99 (2004).

6. J.D. Smith, C.D. Cappa, K.R. Wilson, B.M. Messer, R.C. Cohen, R.J. Saykally. Science 306, 851-53 (2004).

7. T. Head-Gordon, G. Hura. Chem. Rev. 102, 2651-69 (2002).

8. A. K. Soper. J. Phys. Chem. B 115, 14014-22 (2011).

9. A. Nilsson, L.G.M. Pettersson. Chem. Phys. 389, 1-34 (2011).

10. C. Huang et al. Proc. Nat. Acad. Sci. 106, 15214-18 (2009).

11. G.N.I. Clark, C.D. Cappa, J.D. Smith, R.J. Saykally, T. Head-Gordon. Proc. Nat. Acad. Sci. 107, 14003-07 (2010).

12. F. Sedlmeier, D. Horinek, R. R. Netz. J. Am. Chem. Soc. 133, 1391-98 (2011).

13. G.N.I. Clark, C.D. Cappa, J.D. Smith, R.J. Saykally, T. Head-Gordon. 108, 1415-1433 (2010).

14. T.A. Kesselring, G. Franzese, S.V. Buldyrev, H.J. Herrmann and H.E. Stanley. Scientific Reports 2, 474 (2012).

15. C.J. Benmore, R.T. Hart, Q. Mei, D.L. Price, J. Yarger, C.A. Tulk, D.D. Klug. Phys. Rev. B 72, 132201 (2005).

16. J. Teixeira. Mol. Phys. 110, 249-588 (2012).

17. L.B. Skinner, C Huang, D. Schlesinger, L.G.M. Pettersson, A. Nilsson, C.J. Benmore. J. Chem. Phys. 138, 074506 (2013).

18. J. Neuefeind, C.J. Benmore, J.K.R. Weber, D. Paschek. Mol. Phys. 109, 279-88 (2011).

19. Supplemental Material.

20. L.B. Skinner, C.J. Benmore, J.B. Parise. Nuc. Instum. Meth. A 662, 61-70 (2012).

21. A. Zeidler, P.S. Salmon, H.E. Fischer, J.C. Neuefeind, J.M. Simonson, H. Lemmel, H. Rauch, T.E. Markland. Phys. Rev. Lett. 107 (2011) 145501.

22. A.K. Soper. J. Phys. Condens. Matt. 19, 335206 (2007).

23. J.L.F. Abascal, C. Vega. J. Chem. Phys. 123, 234505 (2005).

24. L. Bosio, S.-H. Chen, J. Teixeira. Phys. Rev. A 27, 1468-75 (1983).

25. G. E. Walrafen, M. R. Fisher, M. S. Hokmabadi, and W.H. Yang. J. Chem. Phys. 85, 6970 (1986). 
26. Y. Maréchal, J. Chem. Phys. 95, 5565-73 (1991).

27. P.L. Geissler. J. Am. Chem. Soc. 127, 14930-35 (2005).

28. C.A. Angell, R.D. Bressel, M. Hemmati, E.J. Sare, J.C. Tucker. Phys. Chem. Chem. Phys. 2, 1559 (2000).

29. R.J. Speedy and C.A. Angell, J. Chem. Phys. 65, 851 (1976).

30. F. Mallamace, C. Corsaro, H.E. Stanley. Scientific Reports 2, 993 (2012). 
Figures.
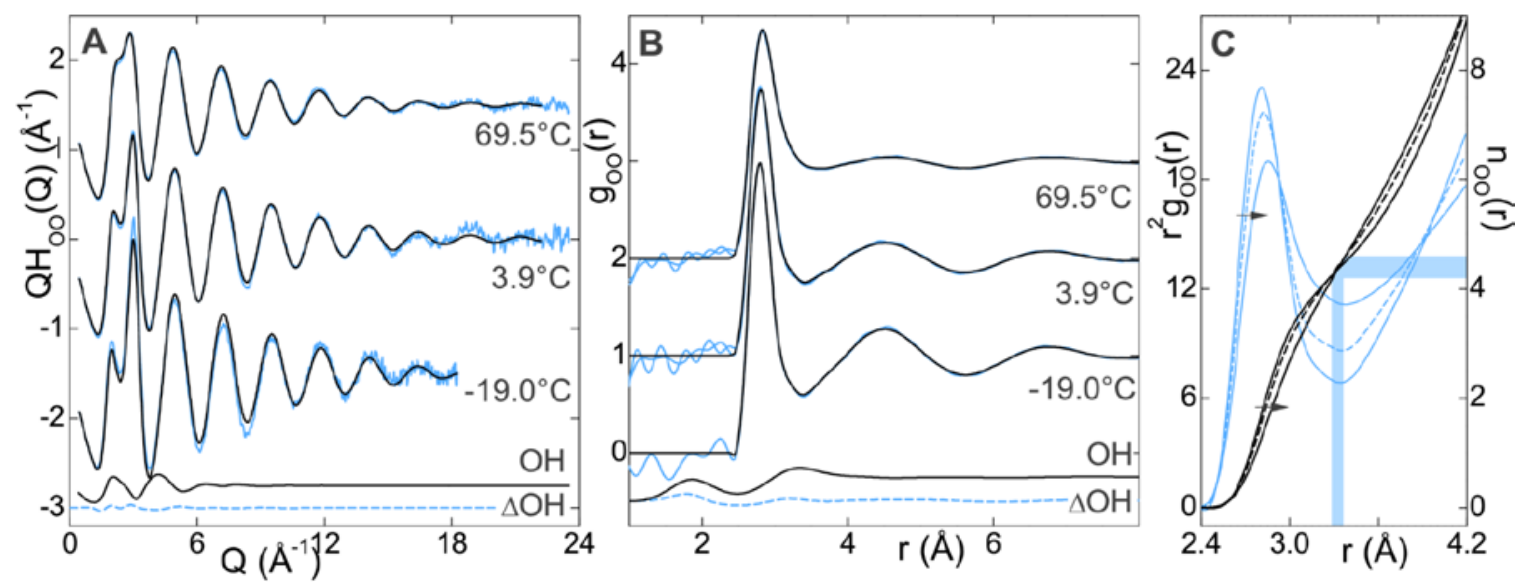

Fig. 1. (A) Oxygen-Oxygen partial structure factors, $H_{O O}(Q)=S_{O o}(Q)-1$, measured using high energy $\mathrm{x}$-ray diffraction and multiplied by $\mathrm{Q}$ to emphasize the high-Q structure. The three upper blue curves are the raw data, and the black lines are backtransforms of the modified $g_{o o}(r)$ patterns (black lines in B). The lower black curve labeled $\mathrm{OH}$ is the $\mathrm{x}$-ray weighted inter-molecular Oxygen-Hydrogen contribution at $22^{\circ} \mathrm{C}$ from neutron diffraction ${ }^{21}$. The lower blue dashed curve labelled $\Delta \mathrm{OH}$ is the change in the x-ray weighted $\mathrm{O}-\mathrm{H}$ contribution over $0-50^{\circ} \mathrm{C}$ temperature difference from TIP4P MD simulations. The intermolecular $\mathrm{H}-\mathrm{H}$ contribution to $\mathrm{x}$-ray pattern (not shown) is $>20$ times smaller than that of $\mathrm{OH}$ ). The $\mathrm{OH}$ curves were subtracted from the $\mathrm{x}$-ray measurement to isolate the $S_{O O}(Q){ }^{17,19}$. The $-19^{\circ} \mathrm{C}$ data was taken from reference ${ }^{18}$. (B) Pair distribution functions generated from the $H_{O O}(\mathrm{Q})$ patterns in (A). The solid blue lines are raw Fourier transforms using maximum Q-values of $18.2 \AA^{-1}$ and $22.2 \AA^{-1}$, shown as a guide to the magnitude of the unphysical Fourier oscillations present in the measurements. The three upper black lines were generated by setting $g_{o o}(r)=0$ for $r<2.4 \AA$, and using a variable modification function ${ }^{17,19}$. The lower $\mathrm{OH}$ and $\Delta \mathrm{OH}$ curves also correspond to those in A. (C) Shows the O-O running coordination number for the three temperatures. The arrows indicate the direction of increasing temperature. There is a temperature-independent isosbestic (crossover) point at 3.30(5) $\AA$ where the coordination number is $4.3(2)$ between -20 to $93^{\circ} \mathrm{C}$. 


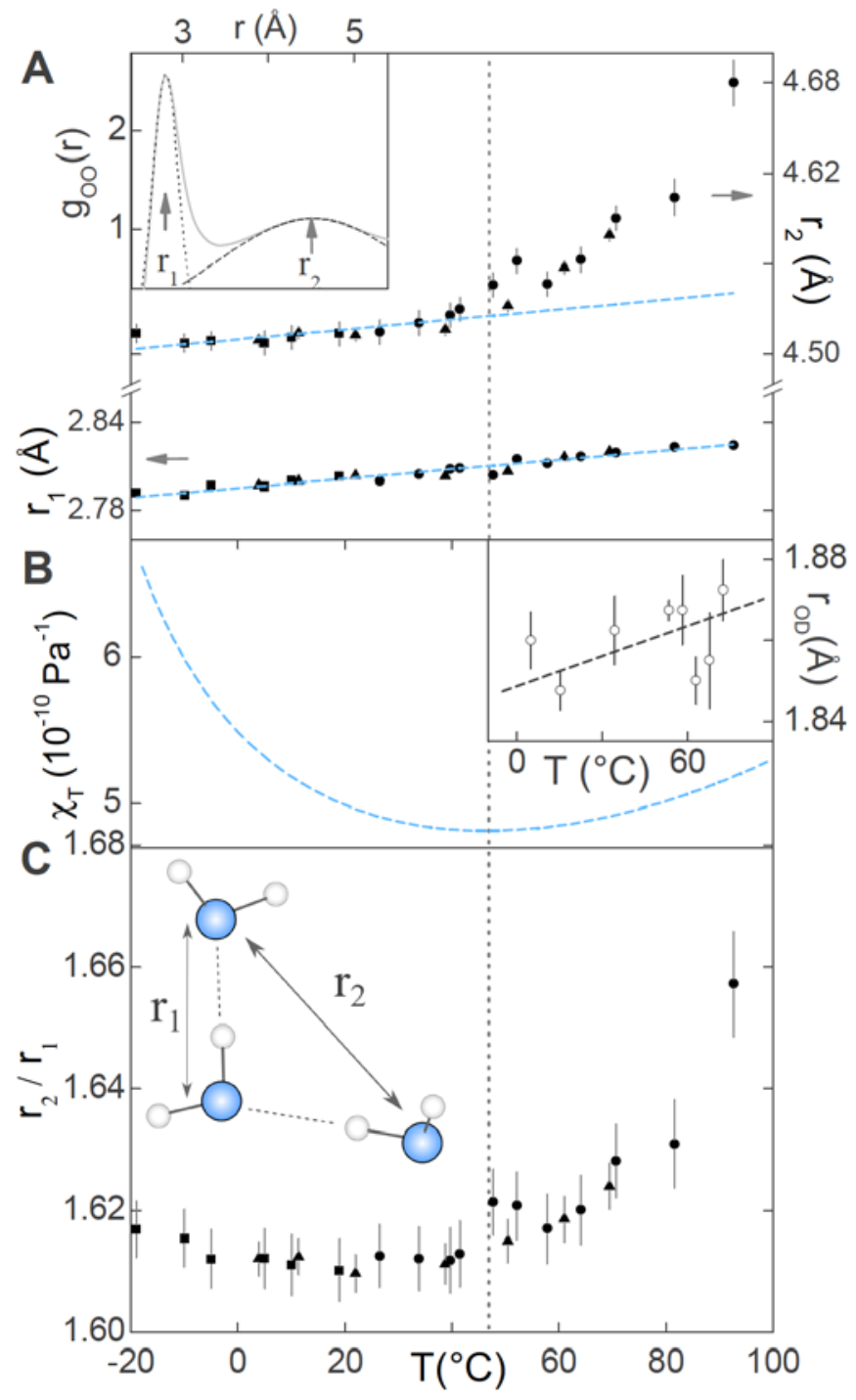

Fig. 2. (A) Positions of the $1^{\text {st }}\left(r_{1}\right)$ and $2^{\text {nd }}\left(r_{2}\right)$ peak maxima in goo $(r)$ obtained from fitting Gaussian functions locally around the maxima (see inset for $22{ }^{\circ} \mathrm{C}$ ). The error in the $r_{1}$ data points is the size of the symbols. The equation of the blue dashed least squares fit line for $r_{1}=0.000320(18) \mathrm{T}+2.7954(8)$ and the line for $r_{2}$ is fitted to $\mathrm{T}<46{ }^{\circ} \mathrm{C}$. (B) The isothermal compressibility of ambient pressure water from reference ${ }^{1}$. Inset: intermolecular O-D peak position, from neutron diffraction $\mathrm{D}_{2}{ }^{18} \mathrm{O}$ minus $\mathrm{D}_{2}{ }^{16} \mathrm{O}$ difference measurements (open circles), and a straight line fit (dashed line). At room temp this $r_{\mathrm{OD}}=1.85(1) \AA$ measurement is in agreement with Zeidler et $a l^{21}$ at room temp $\left(\mathrm{r}_{\mathrm{OD}}=\right.$ $1.83(2) \AA$ ). (C) The $r_{2} / r_{1}$ ratio (1.633 corresponds to the ideal intra-tetrahedral angle). The different black symbols in (A) and (C) represent three independent experimental runs at the same beamline: Acoustically levitated drops ${ }^{18}$ (squares), flowing water streams (triangles), and water in a glass capillary (circles). Notice the nearest neighbor distance expands linearly, whereas the $\mathrm{r}_{2}$ behavior changes above the compressibility minimum, and below the density maximum. 

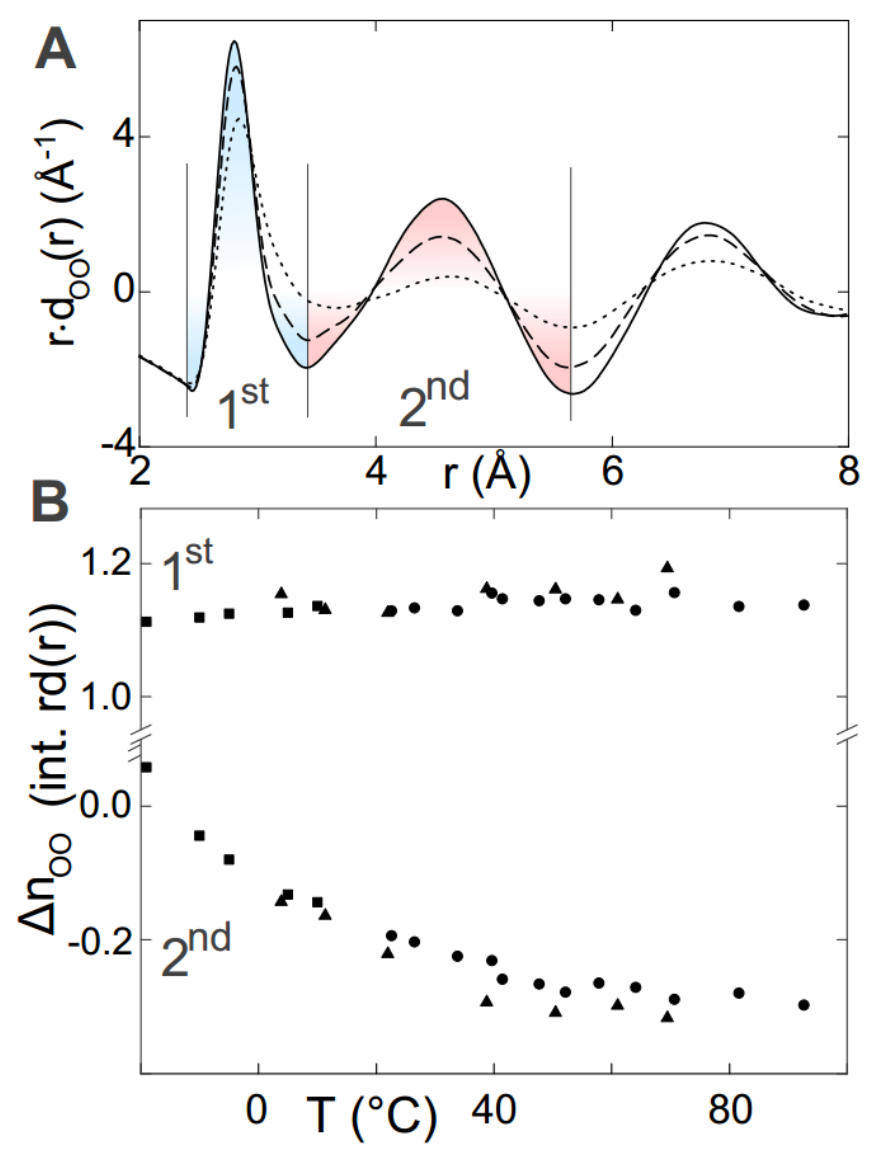

Fig. 3. (A) The $\mathrm{O}-\mathrm{O}$ pair distribution functions at three temperatures: $-19^{\circ} \mathrm{C}$ (solid line), $3.9^{\circ} \mathrm{C}$ (dashed), and $69{ }^{\circ} \mathrm{C}$ (dotted). The areas highlighted in blue are designated $1^{\text {st }}$ neighbor peak $(2.4<r<3.3 \AA)$ and $2^{\text {nd }}$ neighbor peak $(3.3<r<5.6 \AA)$. (B) Shows the integrals of the experimental $r \cdot d(r)$ functions in the two regions versus temperature. The area of the $1^{\text {st }}$ peak is essentially constant. 